\title{
Review of: "Robust Working Memory through Short- Term Synaptic Plasticity"
}

\author{
Christian Huyck ${ }^{1}$ \\ 1 Middlesex University
}

Potential competing interests: The author(s) declared that no potential competing interests exist.

This is a solid paper. I think the importance of short term plasticity is currently recognised,. The author's study on monkeys using single unit recording on delay tasks is a solid addition to the area. However, I found it a bit odd that the simulation used units that were not biologically plausible (ReLU), despite citing work by authors that used spiking neurons and short term potentiation (Lansner). It reminded me of Plaut's work using backprop nets in the 90s. It's not that these simulations are entirely irrelevant, but merely speak to the distributed nature of the computation. Moreover, I didn't see a link to the code or data, which is clearly a flaw. None the less, the read was entirely worthwhile. 